\title{
Erratum to: Development of an auditory emotion recognition function using psychoacoustic parameters based on the International Affective Digitized Sounds
}

\author{
Youngimm Choi $^{1,2} \cdot$ Sungjun Lee ${ }^{1} \cdot$ SungSoo Jung ${ }^{1}$. \\ In-Mook Choi ${ }^{1}$ - Yon-Kyu Park ${ }^{1} \cdot$ Chobok Kim $^{3}$
}

Published online: 28 April 2015

(C) Psychonomic Society, Inc. 2015

Erratum to: Behav Res

DOI 10.3758/s13428-014-0525-4

The second author of the article, Sungjun Lee (lsjun@kriss.re.kr), should be an additional corresponding author.

The online version of the original article can be found at http://dx.doi.org/ 10.3758/s13428-014-0525-4.

$\triangle$ Sungjun Lee

1sjun@kriss.re.kr

$\triangle$ Chobok Kim

ckim@knu.ac.kr

1 Korea Research Institute of Standards and Science, Daejeon, South Korea

2 Department of Psychology, Chungnam National University, Daejeon, South Korea

3 Department of Psychology, Kyungpook National University, 80 Daehak-ro, Buk-gu, Daegu 702-701, South Korea 